
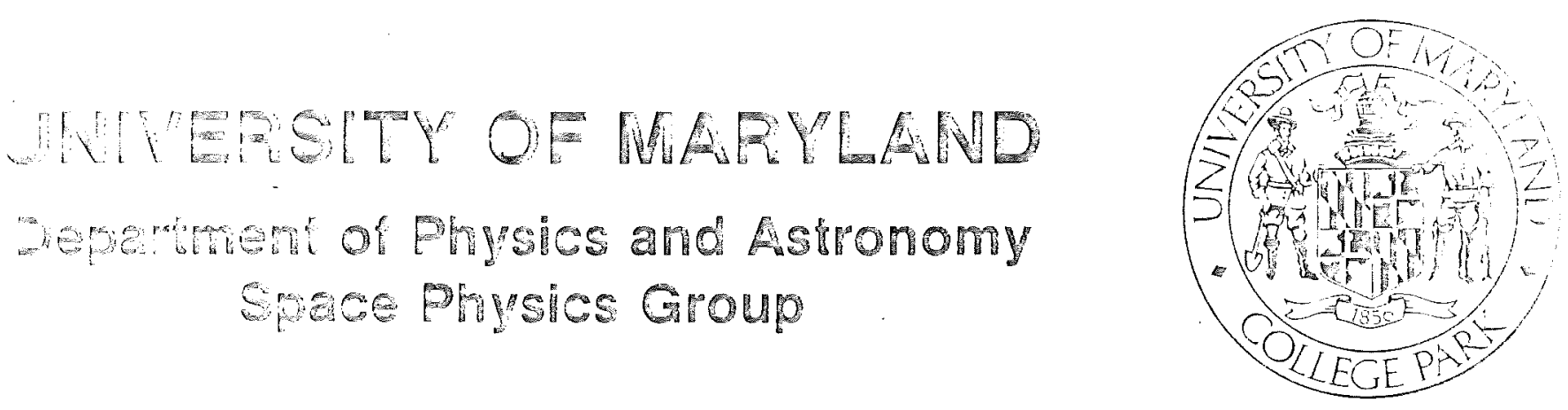

\title{
THE SOLAR, ANOMALOUS, AND MAGNETOSPHERIC PARTICLE EXPLORER (SAMPEX)
}

G. M. Mason ${ }^{a}$, D. N. Baker ${ }^{b}$, J. B. Blakec , L. B. Callis ${ }^{d}$, D. C. Hamilton ${ }^{a}$, D. Hovestadt ${ }^{e}$, B. Klecker ${ }^{e}$, R. A. Mewaldt ${ }^{f}$, M. Scholer ${ }^{e}$, E. C. Stone ${ }^{f}$, and T. T. von Rosenvinge ${ }^{b}$

${ }^{a}$ University of Maryland, College Park, MD 20742

${ }^{b}$ NASA/Goddard Space Flight Center, Greenbelt, MD 20771

${ }^{c}$ Aerospace Corporation, Los Angeles, CA 90009

${ }^{d}$ NASA/Langley Research Center, Hampton, VA 23665

${ }^{e}$ Max-Planck-Institut, 8046 Garching, Germany

${ }^{f}$ California Institute of Technology, Pasadena, CA 91125

PP 92-080

The Hague, The Netherlands

June 25 - July 6, 1990 


\title{
THE SOLAR, ANOMALOUS, AND MAGNETOSPHERIC
} PARTICLE EXPLORER (SAMPEX)

\author{
G. M. Mason ${ }^{a}$, D. N. Baker ${ }^{b}$, J. B. Blake ${ }^{c}$, L. B. Callis ${ }^{d}$, \\ D. C. Hamilton ${ }^{a}$, D. Hovestadt ${ }^{e}$, B. Klecker ${ }^{e}$, R. A. Mewaldt ${ }^{f}$, \\ M. Scholer ${ }^{e}$, E. C. Stone ${ }^{f}$, and T. T. von Rosenvinge ${ }^{b}$ \\ a University of Maryland, College Park, MD 20742 \\ ${ }^{6}$ NASA/Goddard Space Flight Center, Greenbelt, MD 20771 \\ ${ }^{c}$ Aerospace Corporation, Los Angeles, CA 90009 \\ ${ }^{d}$ NASA/Langley Research Center, Hampton, VA 23665 \\ ${ }^{e}$ Max-Planck-Institut, 8046 Garching, FRG \\ ${ }^{f}$ California Institute of Technology, Pasadena, CA 91125
}

\begin{abstract}
The Solar, Anomalous, and Magnetospheric Particle Explorer, SAMPEX, will carry out energetic particle studies of outstanding scientific questions in the fields of space plasma physics, solar physics, magnetospheric and middle atmospheric physics, and cosmic ray physics. SAMPEX will measure the electron and ion composition of energetic particle populations from $\sim 0.4 \mathrm{MeV} /$ nucleon to hundreds of $\mathrm{MeV} /$ nucleon from a zenith-pointing small satellite in near-polar orbit, using a coordinated set of detectors with excellent charge and mass resolution, and with higher sensitivity than previously flown instruments. While over the magnetic poles, the instruments will study the composition of anomalous cosmic rays, solar energetic particles, and galactic cosmic rays. At lower magnetic latitudes, geomagnetic cutoff effects will allow determination of the ionization state of these particles at energies much higher than can be studied from interplanetary spacecraft. At subauroral latitudes, SAMPEX will also observe precipitating relativistic magnetospheric electrons, which undergo important interactions within the middle atmosphere.
\end{abstract}

\section{SCIENTIFIC GOALS}

\section{Anomalous Cosmic Rays}

In the energy range below $\sim 50 \mathrm{MeV} /$ nucleon, there are at least six elements (He, $\mathrm{C}, \mathrm{N}, \mathrm{O}, \mathrm{Ne}$, and $\mathrm{Ar}$ ) whose energy spectra show distinctive increases in flux above the quiet-time galactic cosmic ray spectrum (e.g., /1, 2/). This "anomalous" cosmic ray (ACR) component is generally believed to represent neutral particles that drift into the heliosphere, become ionized by the solar wind or UV radiation, and are then accelerated $/ 3 /$. This model makes a unique prediction: the ACRs will be singly ionized, an assertion for which there is only indirect evidence.

By measuring ACR spectra in a low altitude polar orbit, SAMPEX will directly measure the ACR charge state by using the geomagnetic field as a spectrometer. For example, Figure 1 shows the portions of a polar orbit where $\mathrm{O}^{8+}$ and $\mathrm{O}^{+}$ 
Portion of Orbit when $14-20 \mathrm{MeV} /$ nuc

Oxygen can reach spacecraft ............. $=\mathrm{O}^{8+}(\mathrm{R}-0.4 \mathrm{GV})$

$=-\infty=O^{+}(R \sim 3 G V)$

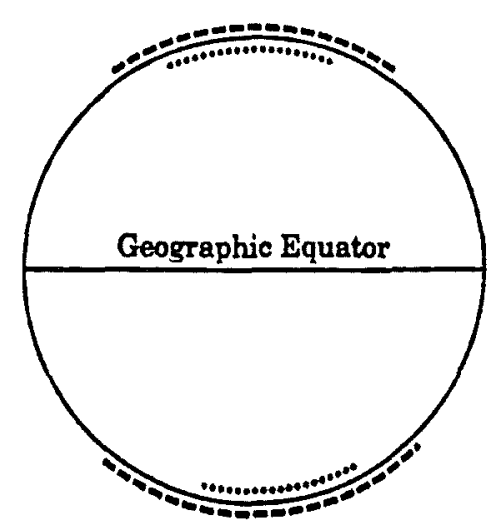

Polar orbit at $0^{\circ}-180^{\circ}$ goographic latitude cutoffs from Shes and Smart, 18hㅡ ICRC, $\underline{3}, 481,1983$.

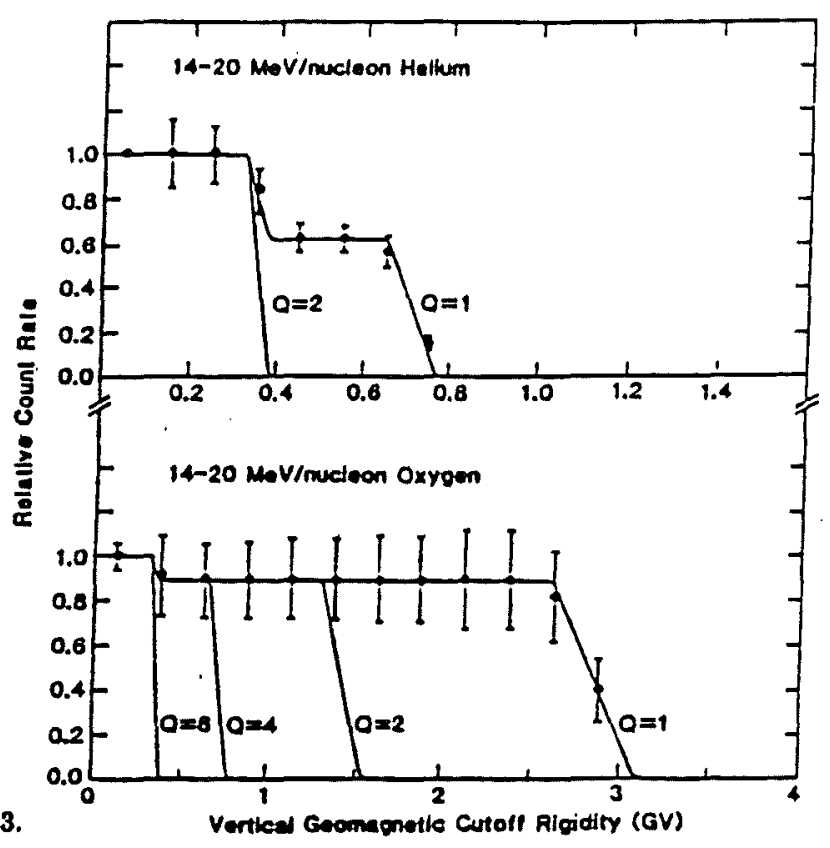

Fig. 1. Access of ACR to SAMPEX.

Fig. 2. ACR fluxes vs. cutoff rigidity.

can reach the spacecraft: the more highly ionized nuclei will be seen during a smaller portion of the orbit since the geomagnetic field is less transparent to them. Indeed, by organizing the particle fluxes by cutoff rigidity, SAMPEX measurements will be able to distinguish amongst a number of possible charge states for ACR nuclei as shown in Figure 2.

If the ACR are indeed singly ionized, then this component represents a direct sample of the local interstellar medium that carries important information about galactic evolution in the solar neighborhood over the time interval since the formation of the solar nebula. In addition, the spectral information obtained by SAMPEX, along with measurements by Voyager, Pioneer, and Ulysses in the outer heliosphere, will provide new insights into the acceleration and transport of these ions.

\section{Solar Energetic Particles - SEP}

Solar flares frequently inject large fluxes of energetic heavy nuclei into the interplanetary medium, where their composition may be determined by direct measurements. The elemental and isotopic composition provide crucial information for understanding the history of solar system material and add new dimensions to the study of solar flare acceleration and propagation processes. High sensitivity spectrometers on SAMPEX will have 1-2 orders of magnitude more collecting power than previous instruments, and thus will determine the composition with sufficient accuracy to make decisive comparisons with other solar system sources. For example, of the few SEP isotopic ratios studied to date, Ne is of special interest since a variety of $\mathrm{Ne}$ isotopic compositions have been observed in the solar system. SEP observations give ${ }^{22} \mathrm{Ne} /{ }^{20} \mathrm{Ne} \simeq 0.12$, significantly different from 


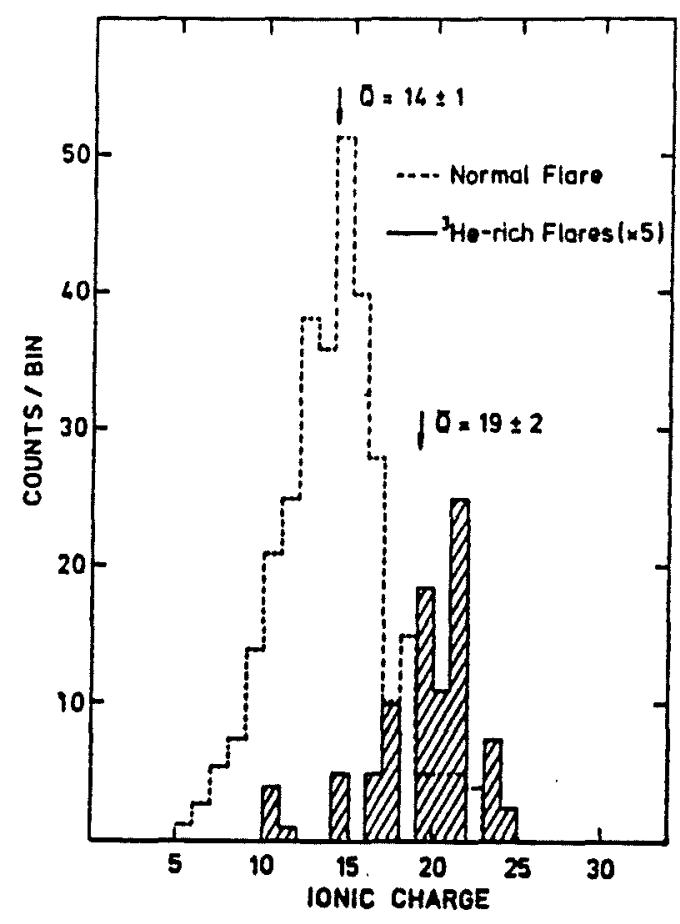

Fig. 3. Charge distribution of iron in the energy range 0.3 to 1.0 $\mathrm{MeV} /$ nucleon obtained for ${ }^{3} \mathrm{He}-$ and Fe-rich solar particle events (cross-hatched histogram) and for one normal solar flare (25-27 Sept. 1978, from /4/).

the solar wind $\left({ }^{22} \mathrm{Ne} /{ }^{20} \mathrm{Ne}=0.073\right)$, and certain meteoritic samples. SAMPEX will measure ${ }^{22} \mathrm{Ne} /{ }^{20} \mathrm{Ne}$ in many solar flares in order to determine whether this is an SEP fractionation effect, or whether other processes such as solar wind fractionation are responsible.

The number of electrons stripped off SEP nuclei is influenced by plasma conditions at the acceleration site; thus, detailed measurements of the charge state distribution of individual ionic species are of importance. $\sim 1 \mathrm{MeV} /$ nucleon SEP from large solar flares show ionization states compatible with coronal temperatures, although it does not seem possible to reconcile the observed distribution with a single equilibrium temperature $/ 5 /$. In addition to large solar flares, there is a class of small, impulsive solar flares with enormous enhancements in the ${ }^{3} \mathrm{He} /{ }^{4} \mathrm{He}$ ratio $\left(10^{3}-10^{4}\right)$ as well as large $(\sim 20)$ enrichments of heavy nuclei such as Fe. Various plasma resonance mechanisms have been proposed to explain these enrichments (e.g., /6/) but many details remain puzzling: for example, particle ionization states in the small flares (see Figure 3 ) show that the heavy nuclei come from hotter sources than normal flares. Using the geomagnetic field, SAMPEX will be able to determine mean SEP ionization states at higher energies than previous measurements, and can distinguish between large percentage differences in charge states (e.g., $\mathrm{Fe}^{14+}$ vs. $\mathrm{Fe}^{20+}$ ) to further constrain possible models for flares.

\section{Precipitating Relativistic Magnetospheric Electrons}

Observations at geosynchronous orbit have found that relativistic $(\gtrsim 1 \mathrm{MeV})$ electron intensities can increase substantially for periods of several days. These enhancements are related to the presence of recurrent solar wind streams, and show a strong solar cycle dependence. Numerical modeling $/ 7 /$ shows that when these electrons precipitate they can cause large energy depositions at 40-60 km 


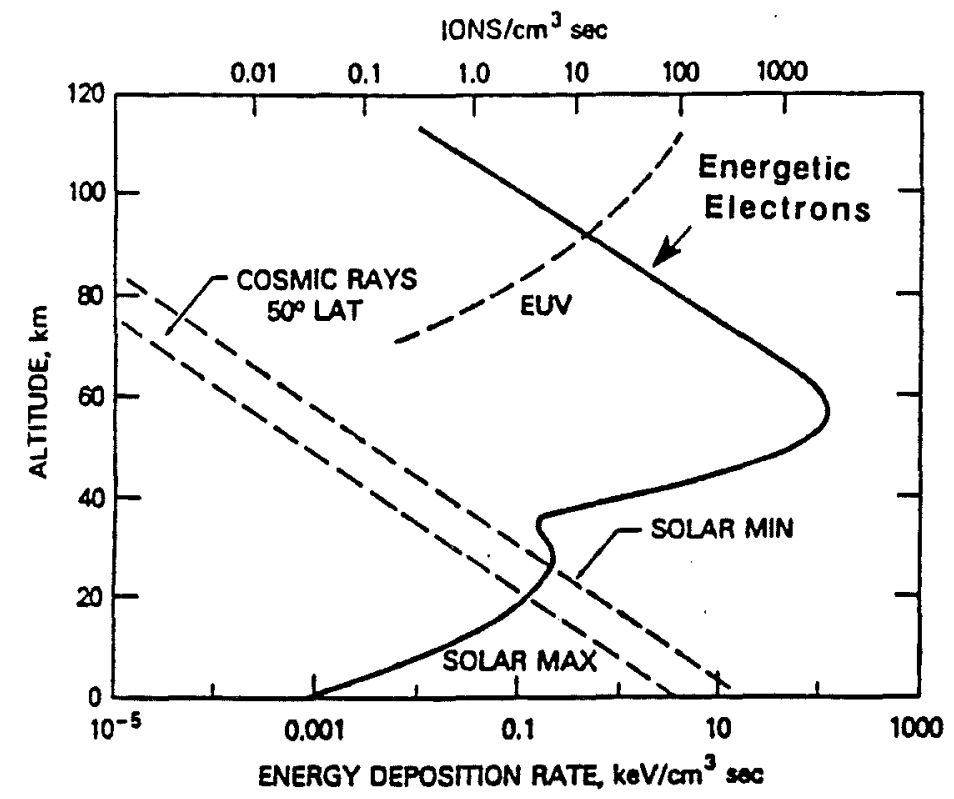

Fig. 4. Atmospheric energy deposition rates at various altitudes for energetic electrons (dark curve), galactic cosmic rays, and solar extreme ultraviolet (EUV) radiation determined from a numerical transport code

altitude in the atmosphere, dominating other ionization sources at these heights (see Figure 4).

Precipitating relativistic electrons may indeed lead to substantial long-term increases in the levels of odd nitrogen compounds $\left(N \mathrm{O}_{x}\right)$ at these heights with an attendant impact on local ozone levels via the reactions $\mathrm{NO}+\mathrm{O}_{3} \rightarrow \mathrm{NO}_{2}+\mathrm{O}_{2}$, and $\mathrm{NO}_{2}+\mathrm{O} \rightarrow \mathrm{NO}+\mathrm{O}_{2}$. It is therefore a critical problem to determine the actual intensity and spatial extent of relativistic electron precipitation. SAMPEX will determine the longitude, latitude, and local time precipitation characteristics for $>1 \mathrm{MeV}$ electrons required for these studies.

\section{Galactic Cosmic Rays}

Galactic cosmic rays are a directly accessible sample of matter from outside the solar system. A spectrometer on SAMPEX will carry out measurements of the isotopic composition of this sample of high energy matter, which contains a record of the nuclear history of cosmic rays including their synthesis in stars and subsequent nuclear interactions with the interstellar gas. Cosmic ray isotope observations have already revolutionized our views of both cosmic ray origin and propagation. As an example, measurements by a number of groups have shown that ${ }^{22} \mathrm{Ne}$ is a factor of at least 3 times more abundant in cosmic ray source material than in the solar system, while the abundances of ${ }^{25} \mathrm{Mg},{ }^{26} \mathrm{Mg},{ }^{29} \mathrm{Si}$, and ${ }^{30} \mathrm{Si}$ are all enhanced by a factor of $\sim 1.5$. The exposure obtained on SAMPEX will make it possible to extend the search for isotopic differences between galactic and solar cosmic ray material to several additional key elements.

\section{INSTRUMENTATION}

The SAMPEX instrumentation includes a complementary set of four high resolution, high sensitivity particle detectors: the Heavy Ion Large Telescope (HILT), the Low Energy Ion Composition Analyzer (LEICA), the Mass Spectrometer Telescope (MAST), and the Proton/Electron Telescope (PET). Prototypes of 


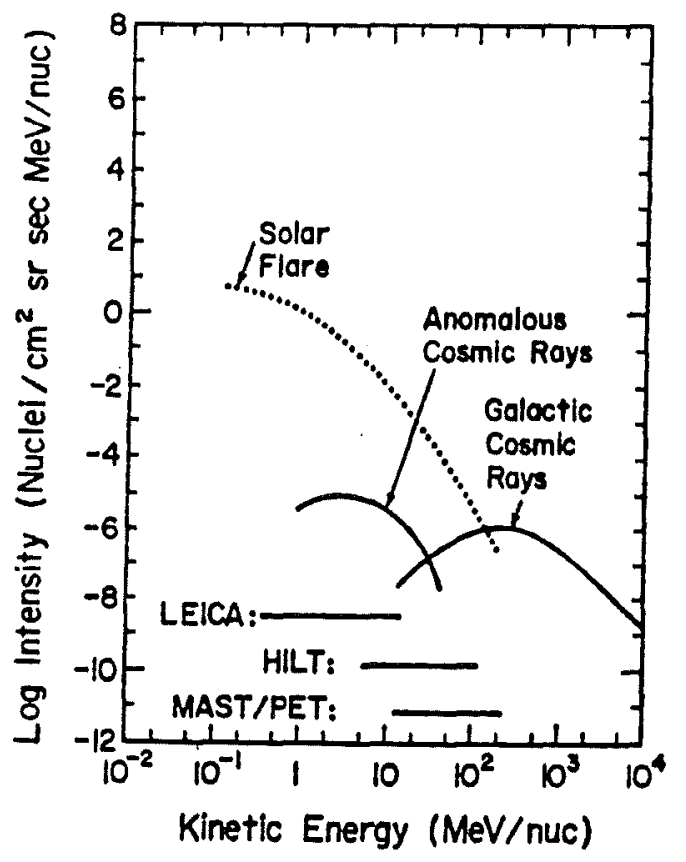

Fig. 5. Spectral coverage of SAMPEX instruments.

TABLE 1 Instrument Performance

LEICA HILT MAST PET

Energy range for

$\begin{array}{lllll}\text { electrons } & - & - & - & 1-30 \mathrm{MeV} \\ \mathrm{H} & 0.75-6 & - & 7-15 & 18-250 \mathrm{MeV} \\ \mathrm{He} & 0.4-6 & 3.9-90 & 7-91 & 18-350 \mathrm{MeV} / \mathrm{n} \\ \mathrm{C} & 0.35-12 & 7.2-160 & 12-210 & 34-120 \mathrm{MeV} / \mathrm{n}^{*} \\ \mathrm{Si} & 0.26-6 & 9.6-177 & 19-345 & 54-195 \mathrm{MeV} / \mathrm{n}^{*} \\ \mathrm{Fe} & 0.16-4 & 11.0-90 & 24-470 & 70-270 \mathrm{MeV} / \mathrm{n}^{*} \\ & & & 1-28 & 1-2(1-28)^{*} \\ \text { range for elements } & 1-28 & 2-28 & 1-28 & 1-2 \\ \text { range for isotopes } & 2-16 & 2 & 1-28 & 0.3-1.6\end{array}$

Charge range for elements

Charge range for isotopes 2-16

Geometrical factor $\left(\mathrm{cm}^{2} \mathrm{sr}\right) \quad 1.0$

65

$0.3-1.6$

* PET commandable low-gain mode

LEICA and HILT flew on the Space Shuttle in August, 1989. MAST and PET were originally approved and under construction for the (cancelled) U.S. spacecraft of the International Solar Polar Mission. Thus, these new instruments are in an advanced state of development and are being readied for SAMPEX quickly and at moderate cost. Table 1 summarizes instrument characteristics. Figure 5 shows the coverage of various energetic particle populations by the SAMPEX ion sensors. 


\section{The Heavy Ion Large Telescope - HILT}

The HILT sensor is a large geometry factor $\left(65 \mathrm{~cm}^{2} \mathrm{sr}\right)$ instrument designed to measure anomalous cosmic rays near the intensity maximum of their spectrum. This will allow statistically accurate spectral and cutoff measurements required for determination of the ACR charge state, as well as the charge states of solar energetic particles. Figure 6 shows a cross section of the sensor. HILT determines particle type by the $d E / d x$ vs. $E$ method; particle trajectory angle is also determined, allowing magnetic cutoff rigidity determination to be done off-line. The proportional-counter and drift chamber are flow-through counters that use isobutane gas. Figure 7 shows the charge resolution of the HILT sensor measured at an accelerator calibration: the resolution is easily sufficient to separate elements of ACR and solar energetic particles.

HILT / SAMPEX CROSS SECTION

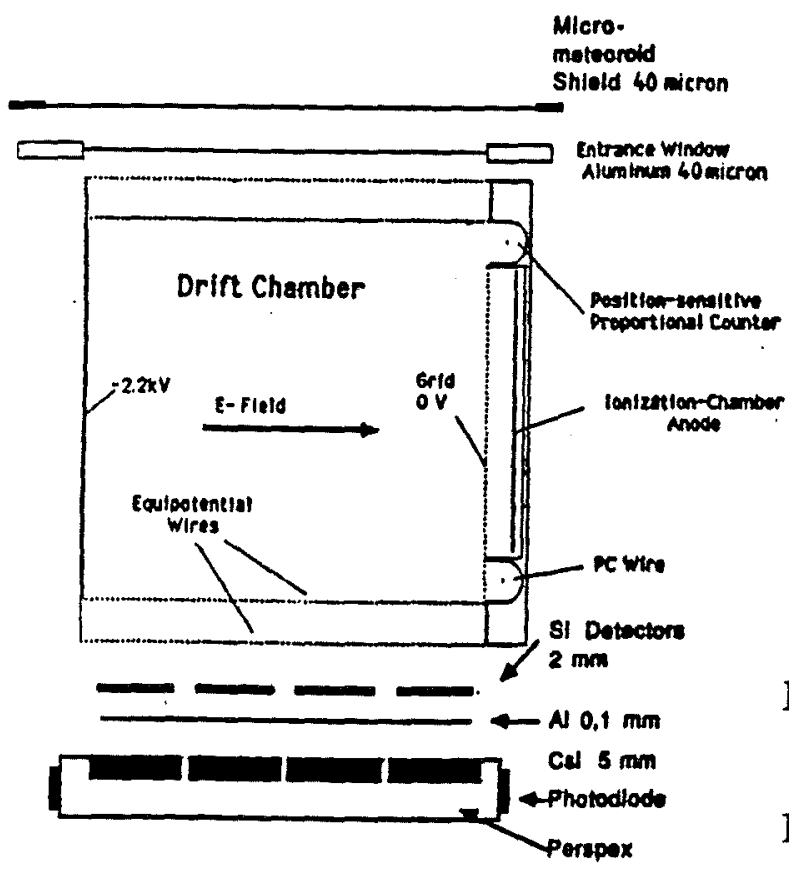

HILTISAMPEX CALIBRATION s2uevinue.

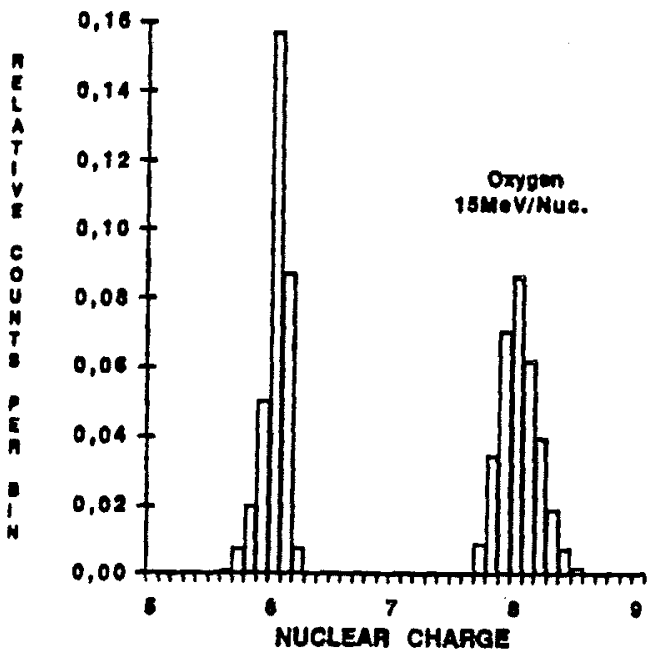

Fig. 6. (left) HILT cross section.

Fig. 7. (above) HILT resolution.

The Low Energy Ion Composition Analyzer - LEICA

LEICA measures elemental and isotopic abundances over the range from about 0.35 to $10 \mathrm{MeV} /$ nucleon. The instrument is a time-of-flight mass spectrometer that identifies incident ion mass $M$ and energy by simultaneously measuring the time-of-flight $t$ and residual kinetic energy $E$ of particles that enter the telescope and stop in one of the array of four Si solid state detectors, as shown in Figure 8. The time of flight is determined by START and STOP pulses from microchannel plate assemblies that detect secondary electrons that are emitted from the entrance foil and the front surface of the solid state detector, respectively, when the ion passes through them. The measured energy $E=1 / 2 M V^{2}$, and the velocity 

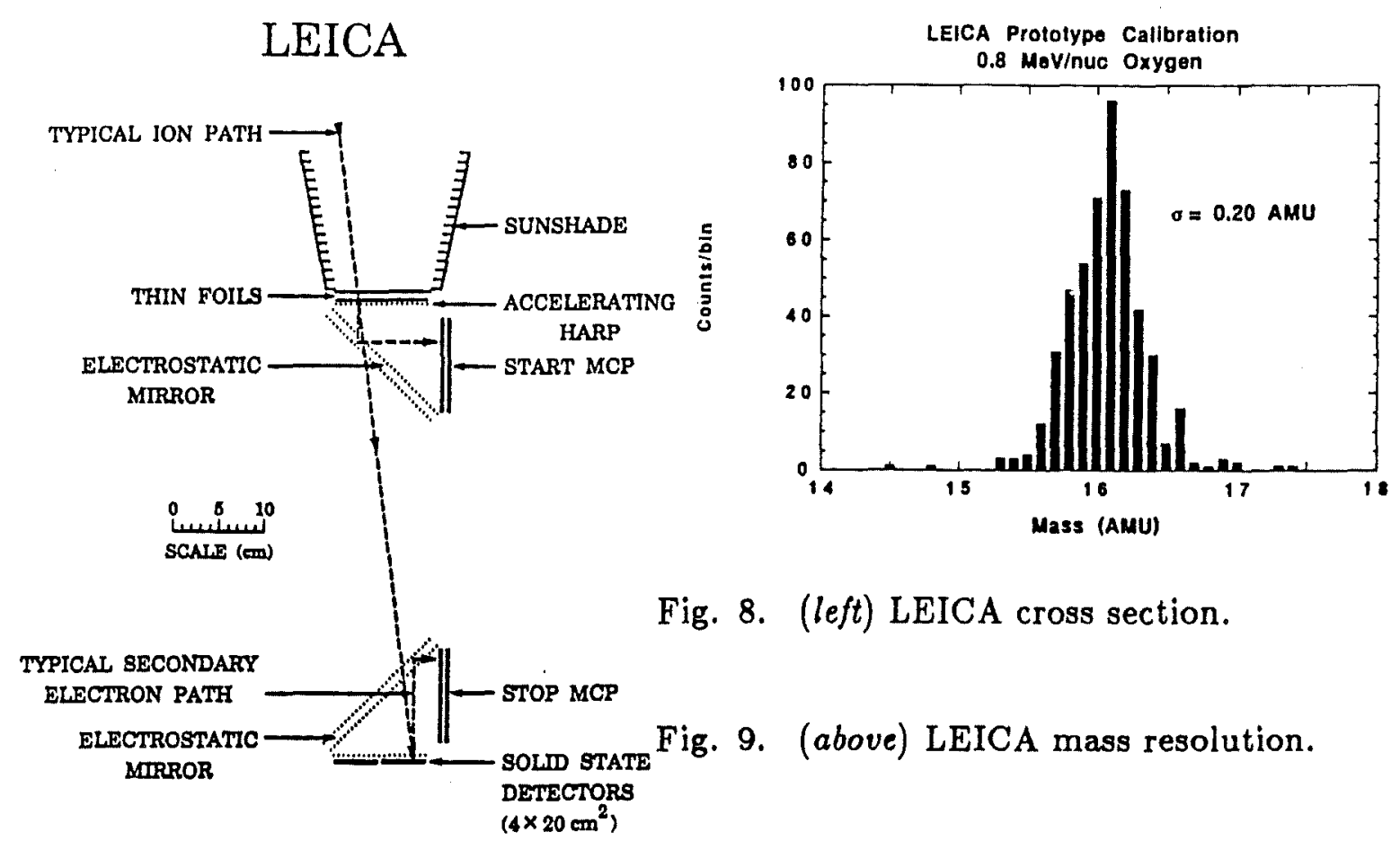

Fig. 8. (left) LEICA cross section.

$V=L / t$ (where $L$ is the path length in the telescope) are combined to yield the mass of the ion $M=2 E(t / L)^{2}$, and the energy per nucleon of the ion. Figure 9 shows the mass resolution of a prototype LEICA telescope.

\section{The MAST/PET Sensors}

The Mass Spectrometer Telescope - MAST. MAST will measure the isotopic composition of elements from $\mathrm{Li}$ to $\mathrm{Ni}$ in the range from $\sim 10 \mathrm{MeV} /$ nucleon to several hundred $\mathrm{MeV} /$ nucleon. MAST consists of a combination of surface barrier and lithium-drifted solid-state detectors as shown in Figure 10. The first four detectors (M1-M4) are one-dimensional position-sensitive detectors (PSDs) that accurately determine particle trajectories in order to permit corrections for pathlength and detector non-uniformities. Isotope identification in MAST is accomplished by the standard $d E / d x$ vs. $E$ technique. The MAST design should achieve a resolution of $\sigma_{m} \leq 0.25 \mathrm{amu}$, sufficient to resolve isotopes with abundance differences of $\sim 100: 1$. Figure 12 shows the excellent resolution obtained with MAST at an accelerator calibration.

The Proton/Electron Telescope - PET. Figure 11 shows the PET system, an all solid state detector telescope designed to complement MAST and HILT by providing measurements of electrons, protons, and helium nuclei. The primary PET analysis mode requires $\mathrm{P} 1$ and $\mathrm{P2}\left(58^{\circ} \mathrm{FOV}\right)$; a wide-angle $90^{\circ} \mathrm{FOV}$ is also available for electrons. PET will also measure $\mathrm{H}$ and $\mathrm{He}$ isotopes, and in its commandable low gain mode it will resolve heavy cosmic ray elements through $Z=28$, thereby providing redundancy for the MAST and HILT sensors. In addition to the coverage indicated in Table 1, PET also includes a special counting rate for 


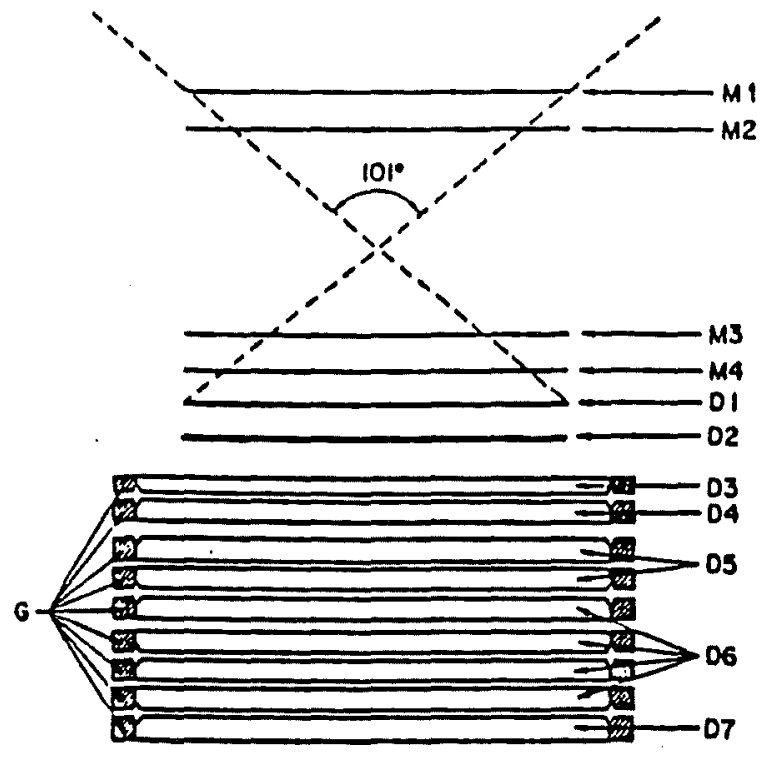

Fig. 10. MAST cross section.

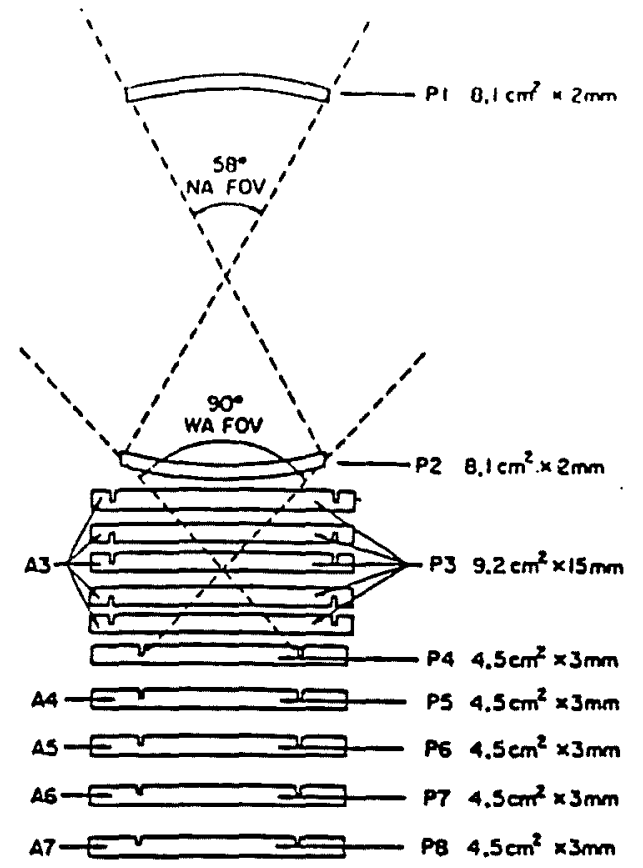

Fig. 11. PET cross section.

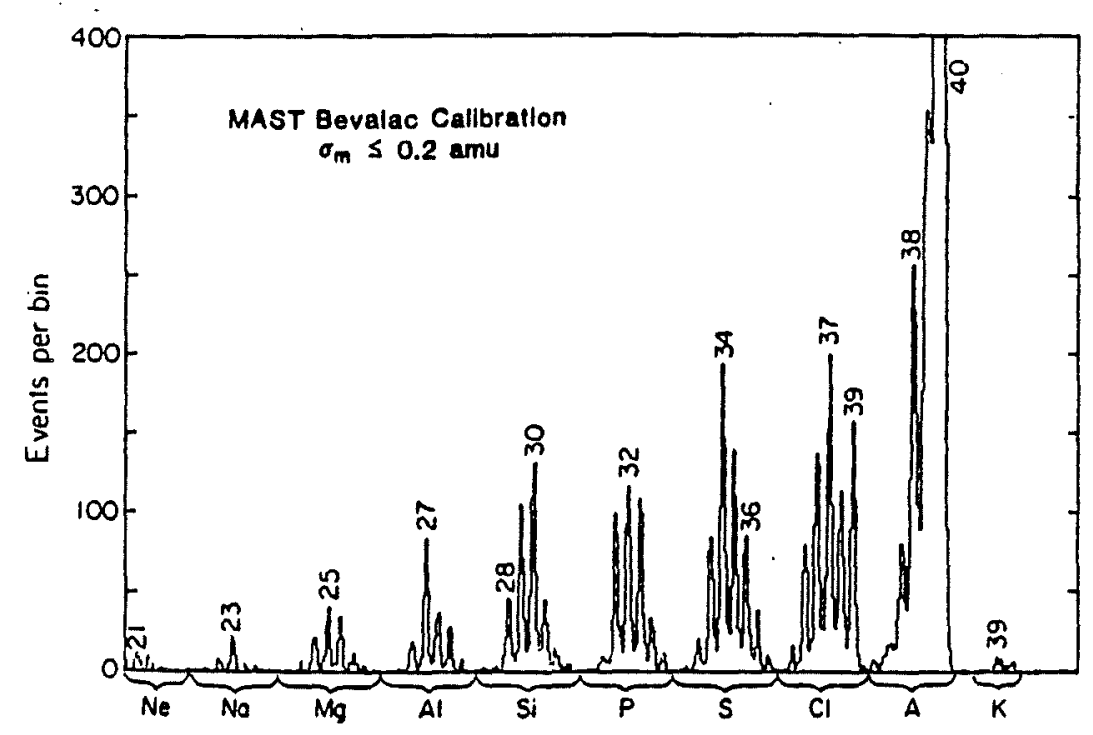

Fig. 12. Mass resolution of MAST obtained at the Bevalac using an ${ }^{40} \mathrm{Ar}$ beam hitting a $\mathrm{CH}_{2}$ target, creating fragmentation products near $\sim 100$ $\mathrm{MeV} /$ nucleon that entered the telescope. Masses of abundant isotopes are indicated.

$>0.4 \mathrm{MeV}$ magnetospheric electrons with $\sim 0.1$ second time resolution.

The Data Processing Unit - DPU. LEICA, HILT, MAST, and PET will be constructed at separate institutions, then integrated with the experiment Data Processing Unit (DPU). This approach requires only a single interface between the SAMPEX experiment and the spacecraft, as shown in Figure 13. 
System Block Dlagram

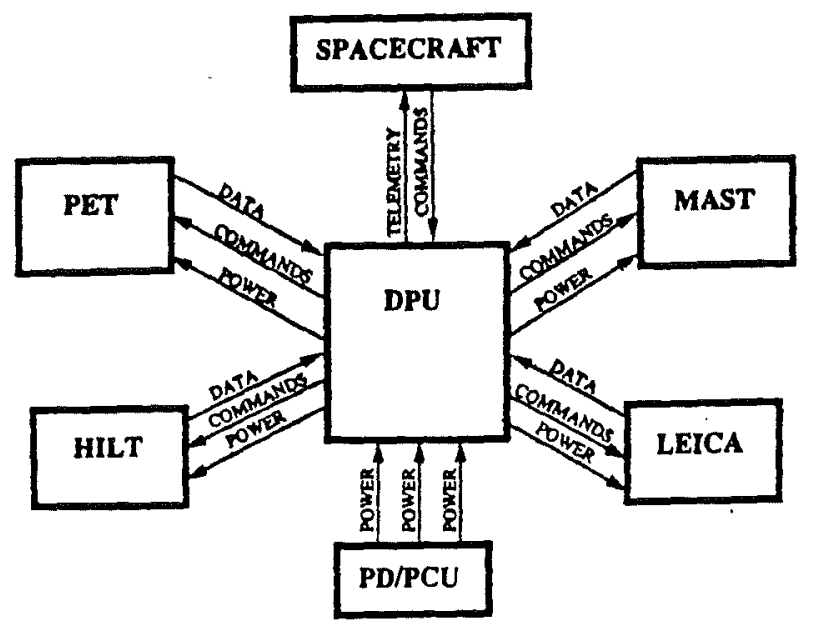

Fig. 13. SAMPEX instrument block diagram showing instruments, the Data Processing Unit (DPU), and interface to spacecraft telemetry and power systems.

\section{SPACECRAFT AND MISSION DESCRIPTION}

SAMPEX scientific objectives require a near polar orbit on a spacecraft that is generally zenith pointing. A scan in local time is part of the mission objectives, hence Sun synchronism is not desired. The orbital altitude is selected to give a minimum lifetime of 3 years in order to observe the anomalous component and relativistic precipitating electrons over a significant portion of the solar cycle, and to be confident of observing numerous solar particle events.

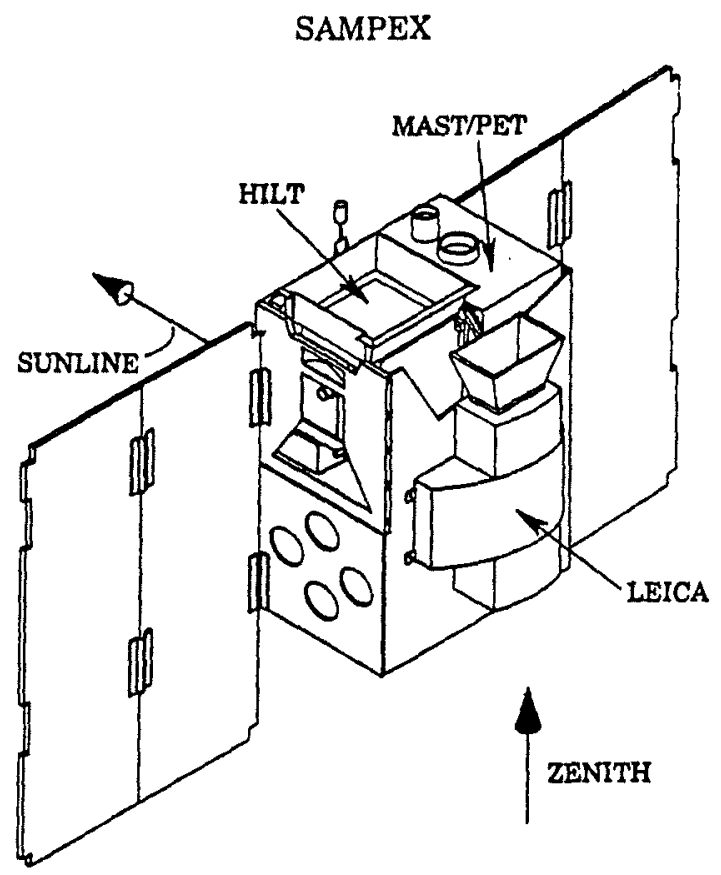

Fig. 14. SAMPEX spacecraft, showing instruments and orientation.

Figure 14 shows the spacecraft layout, and Table 2 summarizes the mission. The spacecraft is a custom design, as required to accommodate already developed in- 
struments in the tight volume constraints of the Scout heat shield. The spacecraft uses momentum-wheel stabilization, with the solar panels always pointing toward the sun in order to achieve the required power. The spacecraft rotates about the sun-pointing vector in such a way as to achieve maximum zenith pointing. For example, for dawn-dusk orbits, the spacecraft rotates once per orbit about the sun-pointing vector, thus achieving zenith pointing for the instruments at all times. For noon-midnight orbits, the spacecraft rotates to be close to zenith pointing near the magnetic poles, then flips over at low magnetic latitudes.

TABLE 2 SAMPEX Mission Description

Spacecraft: built at GSFC

Launch Vehicle: standard Scout

Orbit: $550 \times 675 \mathrm{~km}, 82^{\circ}$ inclination

Launch Date: 1992

Mass: $52 \mathrm{~kg}$ instruments, $118 \mathrm{~kg} \mathrm{~S} / \mathrm{C}+$ margin

Power: $20 \mathrm{~W}$ instruments, $60 \mathrm{~W} \mathrm{~S} / \mathrm{C}$ Total: $80 \mathrm{~W}$

Telemetry: S-band transponder; $5 \mathrm{~W}$ transmitter; $3 \mathrm{MHz}$ downlink; $16 \mathrm{kHz}$ uplink
Launch site: Vandenberg AFB

Orbit average bitrate: $3 \mathrm{kbps}$ Mission Duration: $\geq 3$ years

Total: $170 \mathrm{~kg}$ GaAs solar cells

The instrument DPU sends telemetry packets to the spacecraft data system, where data is stored in a $26 \mathrm{Mb}$ solid state memory. Every 12 hours, the memory is dumped to Wallops or an alternate site. The spacecraft carries a 36-month supply of isobutane for the HILT sensor: there are no other expendables.

Acknowledgements. It is a pleasure to acknowledge the enthusiastic, imaginative, and dedicated support of the Goddard Space Flight Center SMEX project in the design of the SAMPEX spacecraft, which went from selection to Critical Design Review in 15 months. The SAMPEX instrument development is supported by NASA contract NAS5-30704 and by the Bundesministerium für Forschung und Technologie, Federal Republic of Germany, contract $010 \mathrm{C} 9002$.

\section{REFERENCES}

1. G. Gloeckler, Rev. Geophys. Space Phys., 17, 569 (1979).

2. Cummings, A. C., E. C. Stone, and W. R. Webber, Proc. 21st Int'?. Cosmic Ray Conf., 6, 190, 1990.

3. L. A. Fisk, B. Kozlovsky, and R. Ramaty, Ap. J. (Letters), 190, L35 (1974).

4. B. Klecker, D. Hovestadt, G. Gloeckler, F. M. Ipavich, C. Y. Fan, L. A. Fisk, and M. Scholer, Ap. J., 281, 458 (1984)

5. A. Luhn, B. Klecker, D. Hovestadt, G. Gloeckler, F. M. Ipavich, M. Scholer, C. Y. Fan, and L. A. Fisk, Adv. Space Res., 4, No. 2-3, 161 (1984).

6. L. A. Fisk, $A p$. J., 224, 1048 (1978).

7. D. N. Baker, J. B. Blake, D. J. Gorney, P. R. Higbie, R. W. Klebesadel, and J. H. King, Geophys. Res. Letters, 14, 1027 (1987). 